\title{
LOCAL NEWFORMS AND FORMAL EXTERIOR SQUARE $L$-FUNCTIONS
}

\author{
MICHITAKA MIYAUCHI AND TAKUYA YAMAUCHI
}

\begin{abstract}
Let $F$ be a non-archimedean local field of characteristic zero. Jacquet and Shalika attached a family of zeta integrals to unitary irreducible generic representations $\pi$ of $\mathrm{GL}_{n}(F)$. In this paper, we show that Jacquet-Shalika integral attains a certain $L$-function, so called the formal exterior square $L$-function, when the Whittaker function is associated to a newform for $\pi$. By consideration on the Galois side, formal exterior square $L$-functions are equal to exterior square $L$-functions for some principal series representations.
\end{abstract}

\section{INTRODUCTION}

Let $F$ be a non-archimedean local field of characteristic zero and $\mathfrak{o}$ its ring of integers with the maximal ideal $\mathfrak{p}$. Let $\pi$ be an irreducible admissible representation of $\mathrm{GL}_{n}(F)$. Via the local Langlands correspondence, there exists a Weil-Deligne representation $\rho$ of the Weil group $W_{F}$ associated to $\pi$. The exterior square $L$-function of $\pi$ is defined by

$$
L\left(s, \pi, \wedge^{2}\right)=L\left(s, \wedge^{2} \rho\right),
$$

where $L\left(s, \wedge^{2} \rho\right)$ is the $L$-factor of the representation $\wedge^{2} \rho$ of $W_{F}$. We suppose that $\pi$ is unitary and generic. We denote by $\mathcal{W}(\pi, \psi)$ the Whittaker model of $\pi$, and by $\mathcal{C}_{c}^{\infty}\left(F^{m}\right)$ the space of Schwartz functions on $F^{m}$. To give an integral representation of $L\left(s, \pi, \wedge^{2}\right)$, Jacquet and Shalika in [11] introduced a family of zeta integrals of the form $J(s, W, \Phi)$ for $n$ even, and $J(s, W)$ for $n$ odd, where $W \in \mathcal{W}(\pi, \psi)$ and $\Phi \in \mathcal{C}_{c}^{\infty}\left(F^{n / 2}\right)$. In loc. cit., they showed that the integral $J(s, W, \Phi)$ attains $L\left(s, \pi, \wedge^{2}\right)$ when $\pi$ is unramified and $W$ is spherical. The key for unramified computation is the explicit formula for the spherical Whittaker functions given by CasselmanShalika [3] and Shintani [18].

It is natural to ask about ramified representations. Jacquet, Piatetski-Shapiro and Shalika introduced the concept of newforms for generic representations of $\mathrm{GL}_{n}(F)$ in [10], which is an extension of that of spherical vectors for unramified representations. Recently, Matringe [16] and the first author [17] independently gave an explicit formula for Whittaker functions associated to newforms on the diagonal torus. We apply this formula to compute the integral $J(s, W, \Phi)$ when $W$ is associated to a newform.

To state our results, we introduce the notion of the formal exterior square L-functions. For an irreducible admissible representation $\pi$ of $\mathrm{GL}_{n}(F)$, its standard $L$-function can be written as

$$
L(s, \pi)=\prod_{i=1}^{n}\left(1-\alpha_{i} q^{-s}\right)^{-1}, \alpha_{i} \in \mathbf{C},
$$

where $q$ denotes the cardinality of the residue field of $F$. We define the formal exterior square $L$-function of $\pi$ by

$$
\mathscr{L}\left(s, \pi, \wedge^{2}\right)=\prod_{1 \leq i<j \leq n}\left(1-\alpha_{i} \alpha_{j} q^{-s}\right)^{-1}
$$

Date: September 19, 2018.

2010 Mathematics Subject Classification. Primary 22E50, 22E35.

Key words and phrases. local newform, exterior square $L$-function, Rankin-Selberg method. 
It is known that $\mathscr{L}\left(s, \pi, \wedge^{2}\right)$ is equal to $L\left(s, \pi, \wedge^{2}\right)$ for unramified principal series representations, and one may check that $\mathscr{L}\left(s, \pi, \wedge^{2}\right)$ divides $L\left(s, \pi, \wedge^{2}\right)$ in general (Theorem 6.2 (ii)). In this paper, we shall show the following:

Theorem 1.1. Let $\pi$ be a unitary irreducible generic representation of $\mathrm{GL}_{n}(F)$. Suppose that a function $W$ in $\mathcal{W}(\pi, \psi)$ is associated to a newform for $\pi$. Then the integral $J\left(s, W, \Phi_{c}\right)(J(s, W)$ if $n$ is odd) is a constant multiple of $\mathscr{L}\left(s, \pi, \wedge^{2}\right)$, where $c$ is the conductor of $\pi$ and $\Phi_{c}$ is the characteristic function of $\mathfrak{p}^{c} \oplus \cdots \oplus \mathfrak{p}^{c} \oplus\left(1+\mathfrak{p}^{c}\right) \subset F^{n / 2}$.

Theorem 1.1 has several applications. We summarize them comparing the recent progress in this topic. The Jacquet-Shalika integrals attached to a unitary irreducible generic representation $\pi$ span a fractional ideal $I_{\pi}$ of $\mathbf{C}\left[q^{-s}, q^{s}\right]$. It is an important fact that $I_{\pi}$ contains 1 . Due to this, we may define Jacquet-Shalika's exterior square $L$-function $L_{J S}\left(s, \pi, \wedge^{2}\right)$ to be the normalized generator of $I_{\pi}$. Kewat and Raghunathan in [13] have already mentioned that this is implicitly proved by Belt in [1. Theorem 1.1 gives an alternative (and brief) proof of this because it implies that $\mathscr{L}\left(s, \pi, \wedge^{2}\right)$ is contained in $I_{\pi}$. Additionally, Theorem 1.1 says that $\mathscr{L}\left(s, \pi, \wedge^{2}\right)$ divides $L_{J S}\left(s, \pi, \wedge^{2}\right)$. Thus the poles of $\mathscr{L}\left(s, \pi, \wedge^{2}\right)$ are also those of $L_{J S}\left(s, \pi, \wedge^{2}\right)$ (Theorem [3.6). Recently, Kewat and Raghunathan in [13] showed the coincidence of $L_{J S}\left(s, \pi, \wedge^{2}\right)$ and $L\left(s, \pi, \wedge^{2}\right)$ for all the essentially square integrable representations of $\mathrm{GL}_{n}(F)$, and for all the generic representations when $n$ is even. Although Theorem 3.6 is obvious for such representations via arguments on the Galois side (see Theorem 6.2 (ii)), it provides an evidence of the equality of $L_{J S}\left(s, \pi, \wedge^{2}\right)$ and $L\left(s, \pi, \wedge^{2}\right)$ for the odd case.

It is still an open problem to find Whittaker functions which attain exterior square $L$-functions through Jacquet-Shalika integral. We give an example of some principal series representations $\pi$ for which $\mathscr{L}\left(s, \pi, \wedge^{2}\right)$ equals to $L\left(s, \pi, \wedge^{2}\right)$ (Proposition 6.4). Therefore Whittaker newforms attain $L\left(s, \pi, \wedge^{2}\right)$ for such representations.

On the other hand, there is another kind of zeta integrals related to exterior square $L$ functions, introduced by Bump and Friedberg [2]. For an irreducible generic representation $\pi$ of $\mathrm{GL}_{n}(F)$, Bump-Friedberg integral has the form $Z\left(s_{1}, s_{2}, W, \Phi\right)$, where $W \in \mathcal{W}(\pi, \psi)$ and $\Phi \in \mathcal{C}_{c}^{\infty}\left(F^{\lfloor(n+1) / 2\rfloor}\right)$. For Bump-Friedberg integral, we obtain the following

Theorem 1.2. Let $\pi$ be an irreducible generic representation of $\mathrm{GL}_{n}(F)$. Suppose that a function $W$ in $\mathcal{W}(\pi, \psi)$ is associated to a newform for $\pi$. Then the integral $Z\left(s_{1}, s_{2}, W, \Phi_{c}\right)$ is a constant multiple of $L\left(s_{1}, \pi\right) \mathscr{L}\left(s_{2}, \pi, \wedge^{2}\right)$, where $c$ is the conductor of $\pi$ and $\Phi_{c}$ is the characteristic function of $\mathfrak{p}^{c} \oplus \cdots \oplus \mathfrak{p}^{c} \oplus\left(1+\mathfrak{p}^{c}\right) \subset F^{\lfloor(n+1) / 2\rfloor}$.

This paper is organized as follows. In section 2, we define the formal exterior square $L$ functions, and relate them with newforms. We show that Jacquet-Shalika integrals attain the formal exterior square $L$-functions when Whittaker functions are associated to newforms for the even case in section 3, and for the odd case in section 4. We consider Bump-Friedberg integral in section 5. The meaning of the formal exterior square $L$-functions on the Galois side is given in section 6 .

Acknowledgements The first author expresses his appreciation to Taku Ishii for giving an introductory talk on this subject at Tambara Institute of Mathematical Sciences The University of Tokyo, and to Takayuki Oda for inviting him to that conference. The authors would like to thank Tomonori Moriyama for helpful conversations and Yoshi-hiro Ishikawa for his useful comments. The first author is partially supported by JSPS Grant-in-Aid for Scientific Research No.21540017. The second author is partially supported by JSPS Grant-in-Aid for Scientific Research No.23740027. 


\section{Preliminaries}

In this section, after fixing notations, we define formal exterior square $L$-functions for irreducible generic representations of $\mathrm{GL}(n)$, and relate them with newforms.

2.1. Notation. Let $F$ be a non-archimedean local field of characteristic zero, $\mathfrak{o}$ its ring of integers, $\mathfrak{p}$ the maximal ideal in $\mathfrak{o}$, and $\varpi$ a generator of $\mathfrak{p}$. Let $\nu$ denote the valuation on $F$ normalized so that $\nu(\varpi)=1$. We write $|\cdot|$ for the absolute value of $F$ normalized so that $|\varpi|=q^{-1}$, where $q$ stands for the cardinality of the residue field $\mathfrak{o} / \mathfrak{p}$ of $F$. Throughout this paper, we fix a non-trivial additive character $\psi$ of $F$ whose conductor is $\mathfrak{o}$, that is, $\psi$ is trivial on $\mathfrak{o}$ and not trivial on $\mathfrak{p}^{-1}$.

We set $G_{n}=\mathrm{GL}_{n}(F)$. Let $B_{n}$ denote the Borel subgroup of $G_{n}$ consisting of the upper triangular matrices, $T_{n}$ the diagonal torus in $G_{n}$ and $U_{n}$ the unipotent radical of $B_{n}$. We write $\delta_{B_{n}}$ for the modulus character of $B_{n}$. We define a subgroup $T_{n, 1}$ of $T_{n}$ by

$$
T_{n, 1}=\left\{\operatorname{diag}\left(a_{1}, a_{2}, \ldots, a_{n-1}, 1\right) \mid a_{1}, \ldots, a_{n-1} \in F^{\times}\right\} .
$$

We use the same letter $\psi$ for the following character of $U_{n}$ induced from $\psi$ :

$$
\psi(u)=\psi\left(\sum_{i=1}^{n-1} u_{i, i+1}\right), \text { for } u=\left(u_{i j}\right) \in U_{n} .
$$

For an irreducible generic representation $(\pi, V)$ of $G_{n}$, we denote by $\mathcal{W}(\pi, \psi)$ its Whittaker model with respect to $\psi$.

2.2. Formal exterior square $L$-functions. Let $\pi$ be an irreducible generic representation of $G_{n}$. We denote by $L(s, \pi)$ the $L$-factor of $\pi$ defined in [4]. Since the degree of $L(s, \pi)$ is equal to or less than $n$, we can write $L(s, \pi)$ as

$$
L(s, \pi)=\prod_{i=1}^{n}\left(1-\alpha_{i} q^{-s}\right)^{-1}, \alpha_{i} \in \mathbf{C} .
$$

Here we allow the possibility that $\alpha_{i}=0$.

We define the formal exterior square $L$-function of $\pi$ by

$$
\mathscr{L}\left(s, \pi, \wedge^{2}\right)=\prod_{1 \leq i<j \leq n}\left(1-\alpha_{i} \alpha_{j} q^{-s}\right)^{-1} .
$$

We say that $\pi$ is unramified if $\pi$ has a non-zero $\operatorname{GL}_{n}(\mathfrak{o})$-fixed vector. Suppose that $\pi$ is unramified. Then $\mathscr{L}\left(s, \pi, \wedge^{2}\right)$ coincides with the exterior square $L$-function $L\left(s, \pi, \wedge^{2}\right)$ of $\pi$ defined through the local Langlands correspondence ([11]).

Proposition 2.2. Suppose that $\pi$ is an irreducible, essentially square integrable representation of $G_{n}$. Then we have

$$
\mathscr{L}\left(s, \pi, \wedge^{2}\right)=1 .
$$

Proof. If $\pi$ is an irreducible, essentially square integrable representation of $G_{n}$, then the degree of $L(s, \pi)$ is equal to or less than 1 (see [9]). The assertion follows immediately from this.

Let $X=\left(X_{1}, \ldots, X_{n}\right)$ be an $n$-tuple of indeterminates. If $f \in \mathbf{Z}^{n}$ satisfies $f_{1} \geq \ldots \geq f_{n} \geq 0$, then we denote by $s_{f}(X)$ the Schur polynomial in $X_{1}, \ldots, X_{n}$ associated to $f$, that is,

$$
s_{f}(X)=\frac{\left|\left(X_{j}^{f_{i}+n-i}\right)_{1 \leq i, j \leq n}\right|}{\prod_{1 \leq i<j \leq n}\left(X_{i}-X_{j}\right)}
$$

(see [15] Chapter I, section 3). Since $s_{f}(X)$ is a symmetric polynomial, the number $s_{f}(\alpha)=$ $s_{f}\left(\alpha_{1}, \ldots, \alpha_{n}\right)$ is independent of the ordering of $\alpha_{1}, \ldots, \alpha_{n}$ in (2.1). 
Until the end of this subsection, we assume that the degree $k$ of $L(s, \pi)$ is less than $n$. Then we can take $\alpha_{1}, \ldots, \alpha_{n}$ so that $\alpha_{1} \alpha_{2} \cdots \alpha_{k} \neq 0$ and $\alpha_{k+1}=\alpha_{k+2}=\cdots=\alpha_{n}=0$. Thus we have

$$
\mathscr{L}\left(s, \pi, \wedge^{2}\right)=\prod_{1 \leq i<j \leq k}\left(1-\alpha_{i} \alpha_{j} q^{-s}\right)^{-1} .
$$

Lemma 2.3. With the above notations, if $s_{f}(\alpha) \neq 0$, then we have $f_{k+1}=f_{k+2}=\cdots=f_{n}=0$ and $s_{f}(\alpha)=s_{\left(f_{1}, \ldots, f_{k}\right)}\left(\alpha_{1}, \ldots, \alpha_{k}\right)$.

Proof. The assertion follows from the equations

$$
s_{f}(\alpha)=\left(\alpha_{1} \cdots \alpha_{n}\right)^{f_{n}} s_{\left(f_{1}-f_{n}, \ldots, f_{n-1}-f_{n}, 0\right)}(\alpha)
$$

and

$$
s_{\left(f_{1}, \ldots, f_{n-1}, 0\right)}\left(\alpha_{1}, \ldots, \alpha_{n-1}, 0\right)=s_{\left(f_{1}, \ldots, f_{n-1}\right)}\left(\alpha_{1}, \ldots, \alpha_{n-1}\right)
$$

For $f=\left(f_{1}, \ldots, f_{k}\right) \in Z^{k}$ which satisfies $f_{1} \geq \ldots \geq f_{k} \geq 0$, we denote by $r(f)=r\left(f_{1}, \ldots, f_{k}\right)$ the irreducible representation of $\mathrm{GL}_{k}(\mathbf{C})$ with dominant weight $f$. Set $A=\operatorname{diag}\left(\alpha_{1}, \ldots, \alpha_{k}\right)$. Then $\operatorname{tr}(r(f) A)$ equals to $s_{f}\left(\alpha_{1}, \ldots, \alpha_{k}\right)([15])$. By the results in [11] subsection 2.2, for $k=2 h$, we have

$$
\mathscr{L}\left(s, \pi, \wedge^{2}\right)=\sum_{l \geq 0} q^{-l s} \sum_{f_{1}+f_{2}+\cdots+f_{h}=l} s_{\left(f_{1}, f_{1}, f_{2}, f_{2}, \ldots, f_{h}, f_{h}\right)}\left(\alpha_{1}, \ldots, \alpha_{k}\right),
$$

and for $k=2 h+1$, we get

$$
\mathscr{L}\left(s, \pi, \wedge^{2}\right)=\sum_{l \geq 0} q^{-l s} \sum_{f_{1}+f_{2}+\cdots+f_{h}=l} s_{\left(f_{1}, f_{1}, f_{2}, f_{2}, \ldots, f_{h}, f_{h}, 0\right)}\left(\alpha_{1}, \ldots, \alpha_{k}\right),
$$

where it is understood that the sum is taken for $f_{1} \geq f_{2} \geq \ldots \geq f_{h} \geq 0$. By Lemma 2.3. one can observe that if $n=2 m$, then

$$
\mathscr{L}\left(s, \pi, \wedge^{2}\right)=\sum_{l \geq 0} q^{-l s} \sum_{f_{1}+\cdots+f_{m-1}=l} s_{\left(f_{1}, f_{1}, \ldots, f_{m-1}, f_{m-1}, 0,0\right)}\left(\alpha_{1}, \ldots, \alpha_{n}\right),
$$

and if $n=2 m+1$, then

$$
\mathscr{L}\left(s, \pi, \wedge^{2}\right)=\sum_{l \geq 0} q^{-l s} \sum_{f_{1}+f_{2}+\cdots+f_{m}=l} s_{\left(f_{1}, f_{1}, f_{2}, f_{2}, \ldots, f_{m}, f_{m}, 0\right)}\left(\alpha_{1}, \ldots, \alpha_{n}\right) .
$$

2.3. Newforms. Put $K_{n, 0}=\mathrm{GL}_{n}(\mathfrak{o})$. For each positive integer $r$, let $K_{n, r}$ be the subgroup of $K_{n, 0}$ consisting of the elements $k=\left(k_{i j}\right)$ in $K_{n, 0}$ which satisfy

$$
\left(k_{n 1}, k_{n 2}, \ldots, k_{n n}\right) \equiv(0,0, \ldots, 0,1) \quad\left(\bmod \mathfrak{p}^{r}\right) .
$$

For any integer $r$, let $\Phi_{r}$ denote the characteristic function of $\mathfrak{p}^{r} \oplus \cdots \oplus \mathfrak{p}^{r} \oplus\left(1+\mathfrak{p}^{r}\right) \subset F^{n}$. The following lemma determines the support of the function $g \in G_{n} \mapsto \Phi_{r}\left(e_{n} g\right)$, where $e_{n}=$ $(0,0, \ldots, 0,1) \in F^{n}$.

Lemma 2.6. Suppose that $r$ is positive. Then we have

$$
\Phi_{r}\left(e_{n} g\right)= \begin{cases}1, & \text { if } g \in U_{n} T_{n, 1} K_{n, r} \\ 0, & \text { otherwise }\end{cases}
$$

for $g \in G_{n}$ 
Proof. Clearly, we have $\Phi_{r}\left(e_{n} g\right)=1$ for $g \in U_{n} T_{n, 1} K_{n, r}$. We shall prove the converse statement. By the Iwasawa decomposition $G_{n}=U_{n} T_{n} K_{n, 0}$, we can write $g$ in $G_{n}$ as $g=u t k$, where $u \in U_{n}$, $t \in T_{n}, k \in K_{n, 0}$. Since the function $g \mapsto \Phi_{r}\left(e_{n} g\right)$ is left $U_{n}$-invariant, we may assume $g=t k$. We write $t=\operatorname{diag}\left(t_{1}, t_{2}, \ldots, t_{n}\right), t_{i} \in F^{\times}$. Suppose that $\Phi_{r}\left(e_{n} t k\right)=1$. Then we obtain $\left|t_{n} k_{n i}\right| \leq q^{-r}$, for $1 \leq i \leq n-1$ and $\left|t_{n} k_{n n}\right|=1$. This implies $\left|k_{n i}\right|<\left|k_{n n}\right|$ for $1 \leq i \leq n-1$. Since $k$ lies in $K_{n, 0}$, we have $\left|k_{n j}\right| \leq 1$ for all $1 \leq j \leq n$, and there exists at least one $j$ such that $\left|k_{n j}\right|=1$. Thus we get $\left|k_{n n}\right|=1$, and hence $\left|t_{n}\right|=1$. So we may assume that $t$ lies in $T_{n, 1}$. In this case, the equation $\Phi_{r}\left(e_{n} t k\right)=\Phi_{r}\left(e_{n} k\right)=1$ precisely means that $k$ belongs to $K_{n, r}$. This completes the proof.

Let $\pi$ be an irreducible generic representation of $G_{n}$. We write $V(r)$ for the space of $K_{n, r^{-}}$ fixed vectors in $V$. Due to [10] (5.1) Théorème (ii), there exists a non-negative integer $r$ such that $V(r) \neq\{0\}$. We denote by $c(\pi)$ the smallest integer with this property. We call $c(\pi)$ the conductor of $\pi$, and $V(c(\pi))$ the space of newforms for $\pi$. By [10] (5.1) Théorème (ii) again, we have

$$
\operatorname{dim} V(c(\pi))=1 .
$$

For simplicity, we say that an element $W$ in $\mathcal{W}(\pi, \psi)$ is a newform if $W$ is the Whittaker function associated to a newform for $\pi$. It follows from [17] Theorem 4.1 and [18] that a newform $W$ in $\mathcal{W}(\pi, \psi)$ is determined by its value at $1 \in G_{n}$.

Proposition 2.8. Let $W$ be a newform in $\mathcal{W}(\pi, \psi)$. If an element $t=\operatorname{diag}\left(t_{1}, \ldots, t_{n}\right) \in T_{n}$ satisfies $W(t) \neq 0$, then we have $\left|t_{i}\right| \leq\left|t_{i+1}\right|$ for all $1 \leq i \leq n-1$.

Proof. By using the central character of $\pi$, we may assume that $t_{n}=1$. Set $f_{i}=\nu\left(t_{i}\right)$, for $1 \leq i \leq n-1$. Since $W$ is $K_{n, c(\pi)}$-invariant, we have $W(t)=W\left(\operatorname{diag}\left(\varpi^{f_{1}}, \ldots, \varpi^{f_{n-1}}, 1\right)\right)$. Hence the proposition follows from [17] Proposition 1.2.

We shall give an integral representation of formal exterior square $L$-functions. We normalize Haar measures on $T_{n}$ and $T_{n, 1}$ so that the volumes of $T_{n} \cap K_{n, 0}$ and of $T_{n, 1} \cap K_{n, 0}$ are one respectively. Note that if the conductor $c(\pi)$ of $\pi$ is positive, then the degree of $L(s, \pi)$ is less than $n([9])$.

Proposition 2.9. Let $\pi$ be an irreducible generic representation of $G_{n}$ whose conductor is positive and let $W$ be the newform in $\mathcal{W}(\pi, \psi)$ such that $W(1)=1$.

(i) Suppose that $n=2 m$. For any element $a=\operatorname{diag}\left(a_{1}, \ldots, a_{m}\right) \in T_{m}$, we set

$$
b=\operatorname{diag}\left(a_{1}, a_{1}, a_{2}, a_{2}, \ldots, a_{m}, a_{m}\right) \in T_{n} .
$$

Then we have

$$
\mathscr{L}\left(s, \pi, \wedge^{2}\right)=\int_{T_{m, 1}} W(b) \delta_{B_{n}}(b)^{-1 / 2}|\operatorname{det} a|^{s} d a .
$$

(ii) Suppose that $n=2 m+1$. For each element $a=\operatorname{diag}\left(a_{1}, \ldots, a_{m}\right) \in T_{m}$, we set $b=$ $\operatorname{diag}\left(a_{1}, a_{1}, a_{2}, a_{2}, \ldots, a_{m}, a_{m}, 1\right) \in T_{n}$. Then we have

$$
\mathscr{L}\left(s, \pi, \wedge^{2}\right)=\int_{T_{m}} W(b) \delta_{B_{n}}(b)^{-1 / 2}|\operatorname{det} a|^{s} d a .
$$

Proof. (i) For $a=\operatorname{diag}\left(a_{1}, \ldots, a_{m-1}, 1\right) \in T_{m, 1}$, we put $f_{i}=\nu\left(a_{i}\right)$, for $1 \leq i \leq m-1$. Since $W$ is fixed by $K_{n, c(\pi)}$, we have

$$
W(b)=W\left(\operatorname{diag}\left(\varpi^{f_{1}}, \varpi^{f_{1}}, \varpi^{f_{2}}, \varpi^{f_{2}}, \ldots, \varpi^{f_{m-1}}, \varpi^{f_{m-1}}, 1,1\right)\right) .
$$

It follows from [17] Theorem 4.1 that

$$
W(b)=\left\{\begin{array}{cl}
\delta_{B_{n}}^{1 / 2}(b) s_{\left(f_{1}, f_{1}, \ldots, f_{m-1}, f_{m-1}, 0,0\right)}\left(\alpha_{1}, \ldots, \alpha_{n}\right), & \text { if } f_{1} \geq \ldots \geq f_{m-1} \geq 0 \\
0, & \text { otherwise }
\end{array}\right.
$$


Thus, (2.4) implies the assertion. Part (ii) follows from (2.5) in a similar fashion.

\section{JaCQuet-Shalika Integral: The EVEn CASE}

We shall prove that Jacquet-Shalika integral attains the formal exterior square $L$-function when the Whittaker function is associated to a newform. In this section, we consider the case when $n=2 m$.

Let $\pi$ be a unitary irreducible generic representation of $G_{n}$. In [11, Jacquet and Shalika introduced a family of zeta integrals which have the form $J(s, W, \Phi), W \in \mathcal{W}(\pi, \psi), \Phi \in \mathcal{C}_{c}^{\infty}\left(F^{m}\right)$, where $\mathcal{C}_{c}^{\infty}\left(F^{m}\right)$ denotes the space of locally constant, compactly supported functions on $F^{m}$ :

$$
J(s, W, \Phi)=\int_{U_{m} \backslash G_{m}} \int_{V_{m} \backslash M_{m}} W\left(\sigma\left(\begin{array}{cc}
1_{m} & Z \\
0 & 1_{m}
\end{array}\right)\left(\begin{array}{cc}
g & 0 \\
0 & g
\end{array}\right)\right) \psi(-\operatorname{tr} Z) d Z \Phi\left(e_{m} g\right)|\operatorname{det} g|^{s} d g,
$$

where $M_{m}=M_{m}(F), V_{m}$ is the space of upper triangular matrices in $M_{m}, e_{m}=(0,0, \ldots, 0,1) \in$ $F^{m}$ and $\sigma$ is the permutation of degree $n=2 m$ given by

$$
\sigma=\left(\begin{array}{cccc|cccc}
1 & 2 & \ldots & m & m+1 & m+2 & \ldots & 2 m \\
1 & 3 & \ldots & 2 m-1 & 2 & 4 & \ldots & 2 m
\end{array}\right) .
$$

By Proposition 1 in [11 section 7 and [1] Proposition 4.3, there exists $\eta>0$ such that the integral $J(s, W, \Phi)$ absolutely converges to a rational function in $q^{-s}$ for $\operatorname{Re}(s)>1-\eta$.

We take Haar measure on $V_{m} \backslash M_{m}$ so that the volume of $V_{m} \backslash\left(V_{m}+M_{m}(\mathfrak{o})\right)$ is one. By using the Iwasawa decomposition $G_{m}=U_{m} T_{m} K_{m, 0}$, we can write an element $g$ in $G_{m}$ as $g=u a k$, $u \in U_{m}, a \in T_{m}, k \in K_{m, 0}$. Then Haar measure $d g$ on $U_{m} \backslash G_{m}$ is decomposed into

$$
\int_{U_{m} \backslash G_{m}} d g=\int_{T_{m}} \delta_{B_{m}}(a)^{-1} d a \int_{K_{m, 0}} d k .
$$

We normalize Haar measures on $T_{m}$ and $K_{m, 0}$ so that the volumes of $T_{m} \cap K_{m, c(\pi)}$ and of $K_{m, c(\pi)}$ are one respectively. Then the following holds:

Theorem 3.1. Let $\pi$ be a unitary irreducible generic representation of $\mathrm{GL}_{2 m}(F)$ and let $W$ be the newform in $\mathcal{W}(\pi, \psi)$ such that $W(1)=1$. Then we have

$$
J\left(s, W, \Phi_{c(\pi)}\right)=\mathscr{L}\left(s, \pi, \wedge^{2}\right),
$$

where $c(\pi)$ is the conductor of $\pi$ and $\Phi_{c(\pi)}$ is the characteristic function of $\mathfrak{p}^{c(\pi)} \oplus \cdots \oplus \mathfrak{p}^{c(\pi)} \oplus$ $\left(1+\mathfrak{p}^{c(\pi)}\right) \subset F^{m}$.

Proof. If $c(\pi)$ is zero, then the theorem follows from Proposition 2 in [11] section 7. We suppose that $c=c(\pi)$ is positive. The proof is quite similar to that for unramified representations. By Lemma 2.6, the map $g \mapsto \Phi_{c}\left(e_{m} g\right)$ is the characteristic function on $U_{m} T_{m, 1} K_{m, c}$. We note that if $k$ belongs to $K_{n, c}$, then $\left(\begin{array}{cc}k & 0 \\ 0 & k\end{array}\right)$ lies in $K_{n, c}$ and fixes $W$. Thus we obtain

$$
J\left(s, W, \Phi_{c}\right)=\int_{T_{m, 1}} \int_{V_{m} \backslash M_{m}} W\left(\sigma\left(\begin{array}{cc}
1_{m} & Z \\
0 & 1_{m}
\end{array}\right)\left(\begin{array}{cc}
a & 0 \\
0 & a
\end{array}\right)\right) \psi(-\operatorname{tr} Z) d Z \delta_{B_{m}}(a)^{-1}|\operatorname{det} a|^{s} d a .
$$

Note that $\left(\begin{array}{cc}1_{m} & Z \\ 0 & 1_{m}\end{array}\right)\left(\begin{array}{ll}a & 0 \\ 0 & a\end{array}\right)=\left(\begin{array}{cc}a & 0 \\ 0 & a\end{array}\right)\left(\begin{array}{cc}1_{m} & a^{-1} Z a \\ 0 & 1_{m}\end{array}\right)$ and $\operatorname{tr} Z=\operatorname{tr}\left(a^{-1} Z a\right)$. So we get

$$
\begin{aligned}
J\left(s, W, \Phi_{c}\right) & =\int_{T_{m, 1}} \int_{V_{m} \backslash M_{m}} W\left(\sigma\left(\begin{array}{cc}
a & 0 \\
0 & a
\end{array}\right)\left(\begin{array}{cc}
1_{m} & Z \\
0 & 1_{m}
\end{array}\right)\right) \psi(-\operatorname{tr} Z) d Z \delta_{B_{m}}(a)^{-2}|\operatorname{det} a|^{s} d a \\
& =\int_{T_{m, 1}} \int_{V_{m} \backslash M_{m}} W\left(\sigma\left(\begin{array}{ll}
a & 0 \\
0 & a
\end{array}\right)\left(\begin{array}{cc}
1_{m} & Z \\
0 & 1_{m}
\end{array}\right) \sigma^{-1}\right) \psi(-\operatorname{tr} Z) d Z \delta_{B_{m}}(a)^{-2}|\operatorname{det} a|^{s} d a .
\end{aligned}
$$


The second equality follows because $\sigma$ belongs to $K_{n, c}$ and fixes $W$. Set $b=\sigma\left(\begin{array}{cc}a & 0 \\ 0 & a\end{array}\right) \sigma^{-1}$. Then we have $b=\operatorname{diag}\left(a_{1}, a_{1}, a_{2}, a_{2}, \ldots, a_{m-1}, a_{m-1}, 1,1\right)$, where $a=\operatorname{diag}\left(a_{1}, a_{2}, \ldots, a_{m-1}, 1\right) \in$ $T_{m, 1}$. By the Iwasawa decomposition $G_{n}=U_{n} T_{n} K_{n, 0}$, we can write

$$
\sigma\left(\begin{array}{cc}
1_{m} & Z \\
0 & 1_{m}
\end{array}\right) \sigma^{-1}=u_{Z} t_{Z} k_{Z}
$$

for $Z \in M_{m}$, where $u_{Z} \in U_{n}, t_{Z} \in T_{n}$ and $k_{Z} \in K_{n, 0}$. Since the $n$-th row of $u_{Z} t_{Z} k_{Z}$ is $(0,0, \ldots, 0,1)$, we can take $t_{Z}$ and $k_{Z}$ so that $t_{Z} \in T_{n, 1}$ and $k_{Z}$ has the $n$-th row $(0,0, \ldots, 0,1)$. This implies that $k_{Z}$ lies in $K_{n, c}$. Hence we obtain

$$
\begin{aligned}
J\left(s, W, \Phi_{c}\right) & =\int_{T_{m, 1}} \int_{V_{m} \backslash M_{m}} W\left(b u_{Z} t_{Z}\right) \psi(-\operatorname{tr} Z) d Z \delta_{B_{m}}(a)^{-2}|\operatorname{det} a|^{s} d a \\
& =\int_{T_{m, 1}} \int_{V_{m} \backslash M_{m}} \psi\left(b u_{Z} b^{-1}\right) W\left(b t_{Z}\right) \psi(-\operatorname{tr} Z) d Z \delta_{B_{m}}(a)^{-2}|\operatorname{det} a|^{s} d a .
\end{aligned}
$$

We write $b=\operatorname{diag}\left(b_{1}, \ldots, b_{n}\right)$ and $t_{Z}=\operatorname{diag}\left(t_{1}, \ldots, t_{n}\right)$. It follows from Proposition 2.8 that if $W\left(b t_{Z}\right) \neq 0$, then we have $\left|b_{i} t_{i}\right| \leq\left|b_{i+1} t_{i+1}\right|$, for $1 \leq i \leq n-1$. So we obtain $\left|t_{i}\right| \leq\left|t_{i+1}\right|$, for $i$ odd. By Proposition 4 in 11 section 5, we have $\left|t_{i}\right| \geq 1$ for $i$ odd, and $\left|t_{i}\right| \leq 1$ otherwise. Thus we get $\left|t_{i}\right|=1$ for all $i$. Proposition 5 in [11] section 5 says that $Z$ lies in $V_{m}+M_{m}(\mathfrak{o})$. So we may take $u_{Z}=t_{Z}=1$ if $W\left(b t_{Z}\right) \neq 0$, and obtain

$$
J\left(s, W, \Phi_{c}\right)=\int_{T_{m, 1}} W(b) \delta_{B_{n}}(b)^{-1 / 2}|\operatorname{det} a|^{s} d a
$$

because $\delta_{B_{m}}(a)^{2}=\delta_{B_{n}}(b)^{1 / 2}$. Now the assertion follows from Proposition 2.9 (i).

Let $I_{\pi}$ be the subspace of $\mathbf{C}\left(q^{-s}\right)$ spanned by $J(s, W, \Phi)$, where $W \in \mathcal{W}(\pi, \psi)$ and $\Phi \in$ $\mathcal{C}_{c}^{\infty}\left(F^{m}\right)$. We shall give an alternative proof of a result by Belt.

Proposition 3.2 ([1] Theorem 2.2, [13]). With the notation as above, $I_{\pi}$ is a fractional ideal of $\mathbf{C}\left[q^{-s}, q^{s}\right]$ which contains 1 .

Proof. By [12] p. 158, $I_{\pi}$ is a $\mathbf{C}\left[q^{-s}, q^{s}\right]$-module. Due to [1] Proposition 4.3, there exists a polynomial $Q(X) \in \mathbf{C}[X]$ such that $Q\left(q^{-s}\right) I_{\pi} \subset \mathbf{C}\left[q^{-s}, q^{s}\right]$. So $I_{\pi}$ is a fractional ideal of $\mathbf{C}\left[q^{-s}, q^{s}\right]$. By Theorem [3.1, $\mathscr{L}\left(s, \pi, \wedge^{2}\right)$ is contained in $I_{\pi}$, so is 1 .

By Proposition 3.2, we can define Jacquet-Shalika's exterior square $L$-function as follows:

Definition 3.3 ([13] Definition 3.4). Due to Proposition 3.2, there exists a polynomial $P(X) \in$ $\mathbf{C}[X]$ such that $P(0)=1$ and $I_{\pi}=\left(1 / P\left(q^{-s}\right)\right)$. We define Jacquet-Shalika's exterior square $L$-function by

$$
L_{J S}\left(s, \pi, \wedge^{2}\right)=\frac{1}{P\left(q^{-s}\right)} .
$$

We give an alternative proof of results on the non-vanishing of Jacquet-Shalika integral in [1] and 12 by using Proposition 3.2.

Proposition 3.4 ([1] Theorem 2.2, [12] Proposition 5.1). Let $\pi$ be a unitary irreducible generic representation of $\mathrm{GL}_{2 m}(F)$. For any $s_{0} \in \mathbf{C}$, there exist $W \in \mathcal{W}(\pi, \psi)$ and $\Phi \in \mathcal{C}_{c}^{\infty}\left(F^{m}\right)$ such that $J\left(s_{0}, W, \Phi\right) \neq 0$.

Proof. By Proposition 3.2, there are $W_{1}, \ldots, W_{k} \in \mathcal{W}(\pi, \psi)$ and $\Phi_{1}, \ldots, \Phi_{k} \in \mathcal{C}_{c}^{\infty}\left(F^{m}\right)$ such that

$$
\sum_{i=1}^{k} J\left(s, W_{i}, \Phi_{i}\right)=1 .
$$

Now the proposition is obvious. 
On the holomorphy of Jacquet-Shalika integral, we obtain the following

Proposition 3.5 (cf. [1] Theorem 2.2). For an irreducible, square integrable representation $\pi$ of $\mathrm{GL}_{2 m}(F)$, there exist $W \in \mathcal{W}(\pi, \psi)$ and $\Phi \in \mathcal{C}_{c}^{\infty}\left(F^{m}\right)$ such that

$$
J(s, W, \Phi)=1 \text {. }
$$

Proof. The proposition follows from Proposition 2.2 and Theorem 3.1 .

Finally, we state a result on poles of $L_{J S}\left(s, \pi, \wedge^{2}\right)$.

Theorem 3.6. Let $\pi$ be a unitary irreducible generic representation of $\mathrm{GL}_{2 m}(F)$. (i) Suppose that $s_{0} \in \mathbf{C}$ is a pole of $L(s, \pi)$ whose order is equal to or more than two. Then $2 s_{0}$ is a pole of $L_{J S}\left(s, \pi, \wedge^{2}\right)$.

(ii) Suppose that $s_{1}$ and $s_{2}$ are two distinct poles of $L(s, \pi)$. Then $s_{1}+s_{2}$ is a pole of $L_{J S}\left(s, \pi, \wedge^{2}\right)$.

Proof. By Theorem [3.1, the formal exterior square $L$-function $\mathscr{L}\left(s, \pi, \wedge^{2}\right)$ is contained in the set $L_{J S}\left(s, \pi, \wedge^{2}\right) \mathbf{C}\left[q^{-s}, q^{s}\right]$. So the theorem follows from the definition of $\mathscr{L}\left(s, \pi, \wedge^{2}\right)$.

\section{JaCquet-Shalika integral: The odD CASE}

In this section, we consider the case when $n=2 m+1$. Let $\pi$ be a unitary irreducible generic representation of $G_{n}$. Then Jacquet-Shalika integral for $\pi$ has the form $J(s, W), W \in \mathcal{W}(\pi, \psi)$ :

$$
J(s, W)=\int_{U_{m} \backslash G_{m}} \int_{V_{m} \backslash M_{m}} W\left(\sigma\left(\begin{array}{ccc}
1_{m} & Z & 0 \\
0 & 1_{m} & 0 \\
0 & 0 & 1
\end{array}\right)\left(\begin{array}{ccc}
g & 0 & 0 \\
0 & g & 0 \\
0 & 0 & 1
\end{array}\right)\right) \psi(-\operatorname{tr} Z) d Z|\operatorname{det} g|^{s-1} d g,
$$

where $\sigma$ is the permutation of degree $n=2 m+1$ given by

$$
\sigma=\left(\begin{array}{cccc|cccc|c}
1 & 2 & \ldots & m & m+1 & m+2 & \ldots & 2 m & 2 m+1 \\
1 & 3 & \ldots & 2 m-1 & 2 & 4 & \ldots & 2 m & 2 m+1
\end{array}\right) .
$$

We normalize Haar measures on $U_{m} \backslash G_{m}$ and $V_{m} \backslash M_{m}$ so that the volumes of $K_{m, 0}$ and of $V_{m} \backslash\left(V_{m}+M_{m}(\mathfrak{o})\right)$ are one respectively. Similar results to those for the even case hold. We shall be brief here.

Theorem 4.1. Let $\pi$ be a unitary irreducible generic representation of $\mathrm{GL}_{2 m+1}(F)$ and let $W$ be the newform in $\mathcal{W}(\pi, \psi)$ such that $W(1)=1$. Then we have

$$
J(s, W)=\mathscr{L}\left(s, \pi, \wedge^{2}\right) .
$$

Proof. Along the lines in the proof of Theorem [3.1, the theorem follows from Proposition 2.9 (ii).

Let $I_{\pi}$ be the subspace of $\mathbf{C}\left(q^{-s}\right)$ spanned by $J(s, W), W \in \mathcal{W}(\pi, \psi)$. As in Proposition 3.2 , the set $I_{\pi}$ is a fractional ideal of $\mathbf{C}\left[q^{-s}, q^{s}\right]$ which contains 1 . Thus we can define Jacquet-Shalika's exterior square $L$-function by

$$
L_{J S}\left(s, \pi, \wedge^{2}\right)=\frac{1}{P\left(q^{-s}\right)},
$$

where $P(X)$ is a polynomial in $\mathbf{C}[X]$ such that $P(0)=1$ and $I_{\pi}=\left(1 / P\left(q^{-s}\right)\right)$.

In the odd case, Schwartz functions are not involved with Jacquet-Shalika integral. So we get the following

Proposition 4.2 (cf. [1] Theorem 6.1). Let $\pi$ be a unitary irreducible generic representation of $\mathrm{GL}_{2 m+1}(F)$ Then there exists $W \in \mathcal{W}(\pi, \psi)$ such that $J(s, W)=1$.

Proof. The proposition follows from the fact that $I_{\pi}$ contains 1 .

We note that Theorem 3.6 holds for the odd case. 


\section{BUMP-FRIEDBERG INTEGRAL}

Bump and Friedberg introduced another kind of Rankin-Selberg type zeta integrals related to exterior square $L$-functions in [2]. In this section, we treat Bump-Friedberg integrals. Set $m=\lfloor(n+1) / 2\rfloor$ and $m^{\prime}=\lfloor n / 2\rfloor$. We define an embedding $J: G_{m} \times G_{m^{\prime}} \rightarrow G_{n}$ by

$$
J\left(g, g^{\prime}\right)_{k, l}=\left\{\begin{array}{cl}
g_{i j}, & \text { if } k=2 i, l=2 j \\
g_{i j}^{\prime}, & \text { if } k=2 i-1, l=2 j-1 \\
0, & \text { otherwise }
\end{array}\right.
$$

for $n$ even, and by

$$
J\left(g, g^{\prime}\right)_{k, l}=\left\{\begin{array}{cl}
g_{i j}, & \text { if } k=2 i-1, l=2 j-1 \\
g_{i j}^{\prime}, & \text { if } k=2 i, l=2 j \\
0, & \text { otherwise }
\end{array}\right.
$$

for $n$ odd.

Let $\pi$ be an irreducible generic representation of $G_{n}$. For $W \in \mathcal{W}(\pi, \psi)$ and $\Phi \in \mathcal{C}_{c}^{\infty}\left(F^{m}\right)$, we define

$$
Z\left(s_{1}, s_{2}, W, \Phi\right)=\int_{U_{m^{\prime}} \backslash G_{m^{\prime}}} \int_{U_{m} \backslash G_{m}} W\left(J\left(g, g^{\prime}\right)\right) \Phi\left(e_{m} g\right)|\operatorname{det} g|^{1 / 2+s_{2}-s_{1}}\left|\operatorname{det} g^{\prime}\right|^{s_{1}-1 / 2} d g d g^{\prime}
$$

if $n$ is even, and

$$
Z\left(s_{1}, s_{2}, W, \Phi\right)=\int_{U_{m^{\prime}} \backslash G_{m^{\prime}}} \int_{U_{m} \backslash G_{m}} W\left(J\left(g, g^{\prime}\right)\right) \Phi\left(e_{m} g\right)|\operatorname{det} g|^{s_{1}}\left|\operatorname{det} g^{\prime}\right|^{s_{2}-s_{1}} d g d g^{\prime}
$$

if $n$ is odd.

We shall show that Bump-Friedberg integral attains the formal exterior square $L$-function when the Whittaker function is associated to a newform.

Theorem 5.1. Let $\pi$ be an irreducible generic representation of $\mathrm{GL}_{n}(F)$ and let $W$ be a non-zero newform in $\mathcal{W}(\pi, \psi)$. Then $Z\left(s_{1}, s_{2}, W, \Phi_{c(\pi)}\right)$ is equal to $L\left(s_{1}, \pi\right) \mathscr{L}\left(s_{2}, \pi, \wedge^{2}\right)$ up to constant, where $\Phi_{c(\pi)}$ is the characteristic function of $\mathfrak{p}^{c(\pi)} \oplus \cdots \oplus \mathfrak{p}^{c(\pi)} \oplus\left(1+\mathfrak{p}^{c(\pi)}\right) \subset F^{m}$.

Proof. If $c(\pi)$ is zero, then the theorem follows from [2] Theorem 3 with easy modification. We assume that $c=c(\pi)$ is positive. Suppose that $n$ is even. Then we have $m=m^{\prime}=n / 2$. For $g, g^{\prime} \in G_{m}$, let

$$
g=u a k, g^{\prime}=u^{\prime} a^{\prime} k^{\prime}
$$

be their Iwasawa decompositions in $G_{m}=U_{m} T_{m} K_{m, 0}$. By Lemma2.6, the function $g \mapsto \Phi_{c}\left(e_{m} g\right)$ is the characteristic function of $U_{m} T_{m, 1} K_{m, c}$. Thus, under a suitable choice of Haar measures, we obtain

$$
\begin{aligned}
Z\left(s_{1}, s_{2}, W, \Phi_{c}\right) & \int_{K_{m, 0}} \int_{T_{m}} \int_{K_{m, c}} \int_{T_{m, 1}} W\left(J\left(a k, a^{\prime} k^{\prime}\right)\right)|\operatorname{det} a|^{1 / 2+s_{2}-s_{1}}\left|\operatorname{det} a^{\prime}\right|^{s_{1}-1 / 2} \delta_{B_{m}}(a)^{-1} \delta_{B_{m}}\left(a^{\prime}\right)^{-1} d a d k d a^{\prime} d k^{\prime} .
\end{aligned}
$$

We have $J\left(a k, a^{\prime} k^{\prime}\right)=J\left(a, a^{\prime}\right) J\left(k, k^{\prime}\right)$ and $J\left(k, k^{\prime}\right) \in K_{n, c}$ since $k$ belongs to $K_{m, c}$. Observe that $\delta_{B_{m}}(a) \delta_{B_{m}}\left(a^{\prime}\right)|\operatorname{det} a|^{-1 / 2}\left|\operatorname{det} a^{\prime}\right|^{1 / 2}=\delta_{B_{n}}\left(J\left(a, a^{\prime}\right)\right)^{1 / 2}$. Hence we obtain

$$
Z\left(s_{1}, s_{2}, W, \Phi_{c}\right)=\int_{T_{m}} \int_{T_{m, 1}} W\left(J\left(a, a^{\prime}\right)\right)|\operatorname{det} a|^{s_{2}-s_{1}}\left|\operatorname{det} a^{\prime}\right|^{s_{1}} \delta_{B_{n}}\left(J\left(a, a^{\prime}\right)\right)^{-1 / 2} d a d a^{\prime} .
$$

Here we note that $W$ is fixed by $K_{n, c}$. By [17] Theorem 4.1, we have

$$
Z\left(s_{1}, s_{2}, W, \Phi_{c}\right)=\sum s_{\left(f_{1}, f_{2}, \ldots, f_{n-1}, 0\right)}(\alpha) q^{-s_{2} \sum_{i=1}^{m-1} f_{2 i}} q^{-s_{1} \sum_{i=1}^{n-1}(-1)^{i+1} f_{i}},
$$


where the summation is over all $\left(f_{1}, f_{2}, \ldots, f_{n-1}\right) \in \mathbf{Z}^{n-1}$ such that $f_{1} \geq f_{2} \geq \ldots \geq f_{n-1} \geq 0$. Due to [2] (3.3), we get

$$
Z\left(s_{1}, s_{2}, W, \Phi_{c}\right)=\left(1-\omega q^{-m s_{2}}\right) L\left(s_{1}, \pi\right) \mathscr{L}\left(s_{2}, \pi, \wedge^{2}\right),
$$

where $\omega=\alpha_{1} \cdots \alpha_{n}$ (for the definition of $\alpha_{i}$, see (2.1)). Since we are assuming that $c(\pi)$ is positive, we have $\omega=0$, so that

$$
Z\left(s_{1}, s_{2}, W, \Phi_{c}\right)=L\left(s_{1}, \pi\right) \mathscr{L}\left(s_{2}, \pi, \wedge^{2}\right),
$$

as required.

We can prove the theorem for the odd case in a similar fashion. So the proof is complete.

\section{The Galois side Via the local Langlands CorRespondence}

In previous sections, we have defined $\mathscr{L}\left(s, \pi, \wedge^{2}\right)$ and shown that it divides $L_{J S}\left(s, \pi, \wedge^{2}\right)$. In this section, we collect the facts corresponding these in the Galois side via the local Langlands correspondence (say LLC for short).

Let $\Omega$ be an algebraically closed field of characteristic zero. Let $F$ be a finite extension of $\mathbf{Q}_{p}$ and $\mathbf{F}_{q}$ be its residue field with the cardinality $q$. Define the inertia group $I_{F}$ by the following exact sequence:

$$
1 \longrightarrow I_{F} \longrightarrow \operatorname{Gal}(\bar{F} / F) \stackrel{\iota}{\longrightarrow} \operatorname{Gal}\left(\overline{\mathbf{F}}_{q} / \mathbf{F}_{q}\right) \longrightarrow 1 .
$$

Take the geometric Frobenius element $\operatorname{Frob}_{q} \in \operatorname{Gal}\left(\overline{\mathbf{F}}_{q} / \mathbf{F}_{q}\right) \simeq \widehat{\mathbf{Z}}$. Then the Weil group $W_{F}$ is defined by the inverse image of $\mathbf{Z}$-span $\operatorname{Frob}_{q}^{\mathbf{Z}}$ by $\iota$, hence we have

$$
1 \longrightarrow I_{F} \longrightarrow W_{F} \stackrel{\iota}{\longrightarrow} \operatorname{Frob}_{q}^{\mathbf{Z}} \simeq \mathbf{Z} \longrightarrow 1 \text {. }
$$

If we fix a lift $\Phi$ of Frob $_{q}$, then $W_{F}$ can be written as $W_{F}=\bigsqcup_{n \in \mathbf{Z}} \Phi^{n} I_{F}$.

A Weil-Deligne representation of $W_{F}$ is a couple of a smooth representation $r=(r, V)$ of $W_{F}$ with a finite dimensional vector space $V$ over $\Omega$ and $N \in \operatorname{End}_{\Omega}(V)$ satisfying the following relation: if $g=\Phi^{n} \sigma, n \in \mathbf{Z}, \sigma \in I_{F}$, then

$$
r(g) N r(g)^{-1}=q^{-n} N .
$$

Let $(r, N)$ be a Weil-Deligne representation of $W_{F}$. By Jordan decomposition, $r(\Phi)$ can be written as the product of a semisimple matrix $T$ and a unipotent matrix $U$. Then for $g=\Phi^{n} \sigma, n \in \mathbf{Z}, \sigma \in I_{F}$ we define

$$
r^{\mathrm{ss}}(g):=T^{n} r(\sigma) .
$$

We call $r^{\mathrm{ss}}$ the $\Phi$-semisimplification of $r$. It is easy to see that a couple $\left(r^{\mathrm{ss}}, N\right)$ forms a WeilDeligne representation. We say that $r$ is $\Phi$-semisimple if $r^{\mathrm{ss}}=r$. For any Weil-Deligne representation $(r, N)$, we define the $L$-function of it by

$$
L(s, r):=\operatorname{det}\left(1-q^{-s} r(\Phi) \mid(\operatorname{Ker} N)^{I_{F}}\right)^{-1} .
$$

For each integer $n \geq 1$, denote by $\mathscr{G}_{F}(n)$ the set of isomorphism classes of $\Phi$-semisimple WeilDeligne representations of dimension $n$ and $\mathscr{A}_{F}(n)$ the set of isomorphism classes of smooth irreducible representations of $\mathrm{GL}_{n}(F)$. Then by [5], [7], there exists a canonical bijective correspondence $\mathscr{G}_{F}(n) \stackrel{\mathrm{LLC}}{\longrightarrow} \mathscr{A}_{F}(n), \rho \mapsto \pi(\rho)$ which is preserving $L$-functions and $\varepsilon$-factors of both sides (see [6], [8]).

Let $\pi$ be an irreducible generic representation of $\mathrm{GL}_{n}(F)$ and $\rho$ be the corresponding WeilDeligne representation via LLC. Recall that by using $L(s, \pi)=\prod_{i=1}^{n}\left(1-\alpha_{i} q^{-s}\right)^{-1}$, we define

$$
\mathscr{L}\left(s, \pi, \wedge^{2}\right)=\prod_{1 \leq i<j \leq n}\left(1-\alpha_{i} \alpha_{j} q^{-s}\right)^{-1}
$$


Theorem 6.2. The following properties are satisfied:

(i) $\mathscr{L}\left(s, \pi, \wedge^{2}\right)=\operatorname{det}\left(1-q^{-s} \rho(\Phi) \mid \wedge^{2}(\operatorname{Ker} N)^{I_{F}}\right)^{-1}$,

(ii) $\mathscr{L}\left(s, \pi, \wedge^{2}\right)$ divides $L\left(s, \wedge^{2} \rho\right)$.

Proof. By definition, (i) is easy to follow. Note that $\wedge^{2} \rho=\left(\wedge^{2} \rho, N \otimes 1+1 \otimes N\right)$. Then we see that $\wedge^{2} \operatorname{Ker} N \subset \operatorname{Ker}(N \otimes 1+1 \otimes N)$ and hence we have

$$
\wedge^{2}(\operatorname{Ker} N)^{I_{F}} \subset\left(\wedge^{2} \operatorname{Ker} N\right)^{I_{F}} \subset \operatorname{Ker}(N \otimes 1+1 \otimes N)^{I_{F}} .
$$

These inclusions are stable under the action of $\Phi$, hence the claim of (ii).

In what follows we study when our $\mathscr{L}\left(s, \pi, \wedge^{2}\right)$ coincides with $L\left(s, \pi, \wedge^{2}\right)$. To do this, we first prove the following:

Lemma 6.3. Let $\pi=\operatorname{Ind}_{B_{n}}^{G_{n}}\left(\chi_{1} \otimes \cdots \otimes \chi_{n}\right)$ be a principal series representation. Then

$$
L\left(s, \pi, \wedge^{2}\right)=\prod_{1 \leq i<j \leq n} L\left(s, \chi_{i} \chi_{j}\right)
$$

Proof. Let $\rho=(\rho, N)$ be the corresponding Weil-Deligne representation via LLC. Then $N=0$ and $\rho: W_{F} \rightarrow W_{F}^{\mathrm{ab}} \simeq F^{\times} \stackrel{\oplus_{i=1}^{n} \chi_{i}}{\longrightarrow} \mathrm{GL}_{n}(\Omega)$ (cf. Theorem 4.2.1 of [14]), hence we have $\wedge^{2} \rho=$ $\oplus_{1 \leq i<j \leq n} \Omega\left(\chi_{i} \chi_{j}\right)$. Then we have that

$$
L\left(s, \pi, \wedge^{2}\right)=L\left(s, \wedge^{2} \rho\right)=\prod_{1 \leq i<j \leq n} L\left(s, \chi_{i} \chi_{j}\right) .
$$

For characters $\chi_{1}, \ldots, \chi_{n}$ of $F^{\times}$we settle the following hypothesis:

(H): for each $i, j(1 \leq i<j \leq n)$, if $\chi_{i}, \chi_{j}$ are ramified, then so is $\chi_{i} \chi_{j}$.

Proposition 6.4. Keep the notations in Lemma 6.3. Under the hypothesis $(H)$, the following equality holds:

$$
\mathscr{L}\left(s, \pi, \wedge^{2}\right)=L\left(s, \pi, \wedge^{2}\right) .
$$

Proof. By the hypothesis $(\mathrm{H})$, the definition of $\mathscr{L}\left(s, \pi, \wedge^{2}\right)$ and Lemma 6.3, we have that

$$
\mathscr{L}\left(s, \pi, \wedge^{2}\right)=\prod_{1 \leq i<j \leq n} L\left(s, \chi_{i} \chi_{j}\right)=L\left(s, \pi, \wedge^{2}\right)
$$

Remark 6.5. Let $\pi=\operatorname{Ind}_{B_{n}}^{G_{n}}\left(\chi_{1} \otimes \cdots \otimes \chi_{n}\right)$ be a unitary principal series representation. Suppose that the characters $\chi_{1}, \ldots, \chi_{n}$ satisfy the hypothesis $(\mathrm{H})$. Then Theorems 3.1 , 4.1 and Proposition 6.4 says that Jacquet-Shalika integral of a newform for $\pi$ attains the exterior square $L$-function $L\left(s, \pi, \wedge^{2}\right)$. A similar result holds for Bump-Friedberg integral because of Theorem 5.1 .

\section{REFERENCES}

[1] D. Belt, On the holomorphy of exterior-square L-functions, preprint, arXiv:1108.2200, 2011.

[2] D. Bump and S. Friedberg, The exterior square automorphic L-functions on GL(n), Festschrift in honor of I. I. Piatetski-Shapiro on the occasion of his sixtieth birthday, Part II (Ramat Aviv, 1989), Israel Math. Conf. Proc., vol. 3, Weizmann, Jerusalem, 1990, pp. 47-65. MR 1159108 (93d:11050)

[3] W. Casselman and J. Shalika, The unramified principal series of p-adic groups. II. The Whittaker function, Compositio Math. 41 (1980), no. 2, 207-231. MR 581582 (83i:22027)

[4] R. Godement and H. Jacquet, Zeta functions of simple algebras, Lecture Notes in Mathematics, Vol. 260, Springer-Verlag, Berlin, 1972. MR 0342495 (49 \#7241) 
[5] M. Harris and R. Taylor, The geometry and cohomology of some simple Shimura varieties, Annals of Mathematics Studies, vol. 151, Princeton University Press, Princeton, NJ, 2001, With an appendix by Vladimir G. Berkovich. MR 1876802 (2002m:11050)

[6] G. Henniart, Caractérisation de la correspondance de Langlands locale par les facteurs $\epsilon$ de paires, Invent. Math. 113 (1993), no. 2, 339-350. MR 1228128 (96e:11078)

[7] _ Une preuve simple des conjectures de Langlands pour $\mathrm{GL}(n)$ sur un corps p-adique, Invent. Math. 139 (2000), no. 2, 439-455. MR 1738446 (2001e:11052)

[8] - Une caractérisation de la correspondance de Langlands locale pour GL(n), Bull. Soc. Math. France 130 (2002), no. 4, 587-602. MR 1947454 (2004d:22013)

[9] H. Jacquet, Principal L-functions of the linear group, Automorphic forms, representations and $L$-functions (Proc. Sympos. Pure Math., Oregon State Univ., Corvallis, Ore., 1977), Part 2, Proc. Sympos. Pure Math., XXXIII, Amer. Math. Soc., Providence, R.I., 1979, pp. 63-86. MR 546609 (81f:22029)

[10] H. Jacquet, I. Piatetski-Shapiro, and J. Shalika, Conducteur des représentations du groupe linéaire, Math. Ann. 256 (1981), no. 2, 199-214. MR 620708 (83c:22025)

[11] H. Jacquet and J. Shalika, Exterior square L-functions, Automorphic forms, Shimura varieties, and $L$ functions, Vol. II (Ann Arbor, MI, 1988), Perspect. Math., vol. 11, Academic Press, Boston, MA, 1990, pp. 143-226. MR 1044830 (91g:11050)

[12] P. K. Kewat, The local exterior square L -function: Holomorphy, non-vanishing and shalika functionals, J. Algebra 347 (2011), 153-172.

[13] P. K. Kewat and R. Raghunathan, On the local and global exterior square L-functions of $\mathrm{GL}_{n}$, preprint, arXiv:1201.4126, 2012.

[14] S. S. Kudla, The local Langlands correspondence: the non-Archimedean case, Motives (Seattle, WA, 1991), Proc. Sympos. Pure Math., vol. 55, Amer. Math. Soc., Providence, RI, 1994, pp. 365-391. MR 1265559 (95d:11065)

[15] I. G. Macdonald, Symmetric functions and Hall polynomials, second ed., Oxford Mathematical Monographs, The Clarendon Press Oxford University Press, New York, 1995, With contributions by A. Zelevinsky, Oxford Science Publications. MR 1354144 (96h:05207)

[16] N. Matringe, Essential Whittaker functions for GL(n) over a p-adic field, preprint, arXiv:1201.5506, 2012.

[17] M. Miyauchi, Whittaker functions associated to newforms for GL $(n)$ over p-adic fields, preprint, arXiv:1201.350\%, 2012.

[18] T. Shintani, On an explicit formula for class-1 "Whittaker functions" on $G L_{n}$ over $\mathfrak{P}$-adic fields, Proc. Japan Acad. 52 (1976), no. 4, 180-182. MR 0407208 (53 \#10991)

Department of Mathematics, Faculty of Science, Kyoto University, Oiwake Kita-Shirakawa SAKYO KYOTO 606-8502 JAPAN

E-mail address: miyauchi@math.kyoto-u.ac.jp

Department of mathematics, Faculty of Educhtion, Kagoshima University, Korimoto 1-20-6 KAGOSHIMA 890-0065 JAPAN

E-mail address: yamauchi@edu.kagoshima-u.ac.jp 